\title{
Feynman operator calculus and singular quantum oscillator
}

\author{
V.S.Popov and M.A.Trusov* \\ ITEP, Moscow, Russia
}

(Dated: June 4, 2008)

\begin{abstract}
New applications of Feynman disentangling method in quantum mechanics are studied and the time-dependent singular oscillator problem is solved in this approach. The important role of representation group theory is discussed in this context.
\end{abstract}

PACS numbers: 03.65.-w, 03.65.Fd

Keywords: Disentangling; Singular quantum oscillator; Transition probabilities

The method of disentangling expressions, containing non-commuting operators (FDM), suggested by Feynman in [1], gave an elegant solution of the harmonic oscillator excitation under the arbitrary time-dependent external force. In further developments the FDM was applied to some other non-stationary quantum mechanical problems, see [2, 3] and [4] for review. And it was shown that the transition matrix elements calculation became much simpler if the FDM was supplied by some considerations from the representation theory of $S U(2)$ or $S U(1,1)$ groups.

In this paper we apply the FDM to a non-quadratic system - singular oscillator with a variable frequency $\omega(t)$. We obtain the self-contained analytic expressions for the transition amplitudes between states with definite quantum numbers (at $t \rightarrow \pm \infty$ ) and calculate the generating functions for transition probabilities.

The problems considered in [1, 3] can be generalized to a model of a singular oscillator with variable frequency:

$$
\begin{gathered}
\hat{H}=\frac{1}{2} p^{2}+\frac{1}{2} \omega(t)^{2} x^{2}+\frac{g}{8 x^{2}}, \\
0<x<+\infty, \quad g=\text { const }, \quad g>-1, \quad \hbar=m=1 .
\end{gathered}
$$

The frequency $\omega(t)$ is an arbitrary real time function. As usual, we propose the boundary conditions:

$$
\omega(t) \rightarrow \omega_{ \pm} \quad \text { at } \quad t \rightarrow \pm \infty
$$

which allows one to define the final and initial states of the oscillator.

It is well known that at a fixed $t$ the instantaneous spectrum of the Hamiltonian (1) is equidistant (see, e.g., [5]):

$$
E_{n}=2 \omega(n-j), \quad j=-\frac{1}{2}-\frac{1}{4} \sqrt{1+g}, \quad n=0,1,2, \ldots
$$

We note further that the operators

$$
J_{1}=\frac{1}{4}(p x+x p), \quad J_{2}=\frac{1}{4}\left(x^{2}-p^{2}\right)-\frac{g}{16 x^{2}}, \quad J_{0}=\frac{1}{4}\left(x^{2}+p^{2}\right)+\frac{g}{16 x^{2}}
$$

satisfy the standard commutation relations of the $s u(1,1)$ algebra:

$$
\left[J_{1}, J_{2}\right]=-i J_{0}, \quad\left[J_{2}, J_{0}\right]=i J_{1}, \quad\left[J_{0}, J_{1}\right]=i J_{2},
$$

and the Hamitonian (1) is a linear combination of operators $J_{0}$ and $J_{2}$ :

$$
H(t)=\left(\omega^{2}(t)+1\right) J_{0}+\left(\omega^{2}(t)-1\right) J_{2} .
$$

The instantaneous eigenfunctions of the Hamiltonian (11) realize the irreducible unitary infinite-dimensional representation of the non-compact $s u(1,1)$ algebra. The corresponding Casimir operator ("angular momentum" squared) proves to be a constant and can be calculated directly:

$$
\mathbf{J}^{2}=J_{0}^{2}-J_{1}^{2}-J_{2}^{2}=\frac{g-3}{16}=j(j+1)
$$

so the weight of this representation is $j$.

*Electronic address: trusov@itep.ru 
For the simplest case $\omega_{+}=\omega_{-}=1$ the initial and the final states are the eigenfunctions of the $J_{0}$ operator:

$$
J_{0} \psi_{n}=\lambda_{n} \psi_{n}, \quad \lambda_{n}=n-j .
$$

According to Ref. [3], the transition amplitude between initial $|m\rangle$ and final $|n\rangle$ states can be expressed in terms of the generalized Wigner function for the irreducible representation of the $s u(1,1)$ algebra with weight $j$ :

$$
w_{m n}=\left|f_{n-j, m-j}^{(j)}\right|^{2}, \quad n, m=0,1,2, \ldots
$$

The latter can be obtained by an analytic continuation of a standard Wigner function for the compact $s u(2)$ algebra; the details of this technique were described in the Appendix A in [3]. In group theory such a method is known as the "Weyl unitary trick" .

A generalization of this approach to the case of unequal initial and final frequencies $\omega_{+} \neq \omega_{-}$is quite obvious, as, according to (5), the transformation from the initial state basis to the final state one is simply a unitary rotation around the axis 1, i.e., an element of the quasi-unitary group $S U(1,1)$. Omitting intermediate calculations (all details can be found in [6]), we derive the following expression for the transition probabilities between states $\left|m, \omega_{-}\right\rangle$and $\left|n, \omega_{+}\right\rangle$(see Eq. (13) from Ref. [3] , for comparison):

$$
w_{m n}=\frac{L !}{((L-S) !)^{2} S !} \frac{\Gamma(L-2 j)}{\Gamma(S-2 j)} \rho^{L-S}(1-\rho)^{-2 j}\left[{ }_{2} F_{1}(-S, L-2 j ; L-S+1 ; \rho)\right]^{2},
$$

where $j$ is defined in (2) and

$$
L=\max (m, n), \quad S=\min (m, n), \quad L-S=|m-n| .
$$

The $\rho$ parameter, $0 \leq \rho<1$, has the same sense as in the well-known problem of transitions in a regular oscillator (see, e.g., [7, 8]) and can be calculated from the classical oscillator equation of motion. The formula (8) furnishes the ultimate answer to the problem given.

The Gauss hypergeometric function ${ }_{2} F_{1}$ in (8) has its first argument being integer and negative (or zero), so it reduces to the Jacobi polynomial. Making necessary transformations, we obtain from (8):

$$
\begin{aligned}
& w_{m n}=\frac{m !}{n !} \frac{\Gamma(n-2 j)}{\Gamma(m-2 j)} \rho^{n-m}(1-\rho)^{-2 j}\left[P_{m}^{(n-m,-2 j-1)}(1-2 \rho)\right]^{2}, \quad n \geq m, \\
& w_{m n}=\frac{n !}{m !} \frac{\Gamma(m-2 j)}{\Gamma(n-2 j)} \rho^{m-n}(1-\rho)^{-2 j}\left[P_{n}^{(m-n,-2 j-1)}(1-2 \rho)\right]^{2}, \quad m \geq n,
\end{aligned}
$$

which coincides with the standard quantum-mechanical result for the transitions in the time-dependent singular oscillator obtained by means of the Shroedinger equation solution (see [9] and references therein). Consider some special cases of (8), (9):

a) The excitation of the ground (vacuum) state: $m=0$,

$$
w_{0 n}=\frac{\Gamma(n-2 j)}{n ! \Gamma(-2 j)} \rho^{n}(1-\rho)^{-2 j} .
$$

In particular, for $g=-1$ we have

$$
j=-\frac{1}{2}, \quad w_{0 n}=\rho^{n}(1-\rho)
$$

This is the utmost value of the $g$ constant, as at $g<-1$ the particle is collapsing into the potential centre [5], so the Hamiltonian is not the self-conjugated operator any longer. It is significant that the expression (11) corresponds to the distribution of charged scalar boson pairs, created in vacuum with a definite momentum $\mathbf{p}$ under the influence of a variable homogeneous electric field [10, 11].

b) At $g=0$ (i.e. $j=-3 / 4$ ) the problem considered corresponds to the regular oscillator with time-dependent frequency on the positive half-axis; in fact, the formulas (9) for this case should coincide for the well-known formulas for the transition probabilities between odd oscillator states via Legendre functions; the latter can be found in [7, 8], for example. And this really takes place, as there exist some relations between Legendre functions and Jacobi polynomials with specific parameter values; see [6] for details. 
The last point to discuss is the generating functions for the transition probabilities of the singular oscillator. For the excitation of the ground state (the most interesting case in practice) it is easy to obtain:

$$
G_{0}(z)=\sum_{n=0}^{\infty} w_{0 n} z^{n}=\left(\frac{1-\rho}{1-\rho z}\right)^{-2 j}, \quad|z|<1 / \rho
$$

while for the transitions from the first excited state $(m=1)$

$$
G_{1}(z)=\sum_{n=0}^{\infty} w_{1 n} z^{n}=\left(\frac{1-\rho}{1-\rho z}\right)^{-2 j+2}\left(-2 j \rho\left(\frac{1-z}{1-\rho}\right)^{2}+z\right), \quad|z|<1 / \rho
$$

The expressions (12) and (13) can be expanded analytically for all the complex plane.

The formulas (12) and (13) are quite convenient to compute various operator mean-values over the transition probability distributions. In particular, for the adiabatic invariant $I=\langle H\rangle / 2 \omega$ one obtains

$$
\frac{I_{+}}{I_{-}}=\frac{\langle n-j\rangle}{m-j}=\frac{1+\rho}{1-\rho}, \quad m=0,1 .
$$

One can show [6] that this expression remains valid for transitions from an arbitrary level.

To summarize, we note that in our paper the transition probabilities of the singular oscillator have been calculated for an arbitrary frequency $\omega(t)$. To solve the problem, the modified FDM was applied and the representation theory for non-compact $s u(1,1)$ algebra has been used. The final result for the transition amplitudes has been presented in a self-contained form, which is rather convenient for further applications. For the transitions from low-lying states the expressions for the generating functions have been derived and the adiabatic invariant variation at the oscillator evolution has been calculated.

\section{Acknowledgments}

This work was partially supported by the Russian Foundation for Basic Research (grant No. 07-02-01116) and by the Ministry of Science and Education of the Russian Federation (grant No. RNP 2.1.1. 1972). One of the authors (M.A.T.) also thanks for partial support the President Grant No. NSh-4961.2008.2 and the President Grant No. MK-2130.2008.2.

[1] R. P. Feynman, Phys. Rev. 84, 108 (1951).

[2] V. S. Popov, Zh.Eksp.Teor.Fiz. 35, 985 (1958).

[3] V. S. Popov, Phys. Lett. A342, 281 (2005).

[4] V. S. Popov, Phys. Usp. 50, 1217 (2007).

[5] L. D. Landau and E. M. Lifshitz, Quantum Mechanics: Non-Relativistic Theory (Pergamon Press, 1977).

[6] M. A. Trusov, in preparation.

[7] V. S. Popov and A. M. Perelomov, Zh.Eksp.Teor.Fiz. 57, 1684 (1969); 56, 1375 (1969).

[8] A. I. Baz, Y. B. Zeldovich, and A. M. Perelomov, Scattering, Reactions and Decay in Nonrelativistic Quantum Mechanics (Israel Program for Scientific Translations, Jerusalem, 1969).

[9] I. A. Malkin and V. I. Man'ko, Dynamical Symmetries and Coherent States of Quantum Systems (Nauka Publishers, Moscow, 1979) [in Russian].

[10] N. B. Narojnyi and A. I. Nikishov, Yad.Fiz. 11, 1072 (1970).

[11] V. S. Popov, Zh.Eksp.Teor.Fiz. 62, 1248 (1972). 